\title{
SECADO INDUSTRIAL DE PINO RADIATA A ALTA TEMPERATURA
}

\author{
Roberto Melo Hermosilla * \\ Martin Pavón Hinrichsen
}

\section{RESUMEN}

Este trabajo proporciona los resultados de los ensayos realizados para determinar las condiciones más favorables de secado de madera aserrada de Pino radiata a alta temperatura.

Se realizaron 16 ensayos de secado a nivel industrial, además de diversos ensayos de laboratorio, variando los principales parámetros que intervienen en el proceso: temperatura de hulbo seco, diferencia psicrométrica, reacondicionado, calentamiento previo, dimensión y espaciamiento de separadores, ancho de las pilas, humedad inicial y humedad final.

Los resultados de los ensayos se evalúan comparando los diferentes programas de secado realizados, en términos de distribución de la humedad final, defectos de secado, contracciones, requerimientos energéticos y costos asociados.

La principal ventaja que presenta el reacondicionado es el alivio de tensiones y la uniformización de la humedad final. Sin embargo, este método produce una coloración indeseable y una elevación considerable del costo específico de secado.

\begin{abstract}
This work is based on applied research, in order to find the best conditions for drying radiata Pine timber, at high temperature.

It includes sixteen test of industrial wood urving and several laboratory tests, varying the principal parameters involved in wood drying process, such as: Dry bulb temperature, wet bulb depression, conditioning, warming up period, thickness of stickers and distance between them, width of stacks, initial moisture content and final moisture content.

The results of the tests are analized based on the comparison between different drying schedules in relation with; final moisture content distribution, drying defects, energy requirements, cost and shrinkages.

The main advantage of conditioning is to reduce tensions and equalize the final moisture content it countenpart an undesired wood colour and increased drying cost.
\end{abstract}

* Los autores: R. Melo H., Ingeniero Civil Mecánico e Industrial, M. Pavón H., Ingeniero Civil Mecánico. División Industrias - Subsede Regional, Instituto Forestal. Barros Arana 121, Piso 3. Concepción - Chile. 


\section{INTRODUCCION}

Durante los últimos años se ha registrado en el país, y especialmente en la Octava Región, un incremento sostenido de la industrialización de la madera de Pino Radiata, de tal modo que el mercado del producto se ha tornado altamente competitivo, obligando a los productores a buscar los medios más eficientes para obtener madera de calidad a bajos costos, especialmente cuando se trata de madera elaborada.

Un adecuado proceso de secado es precisamente una de las técnicas que hace posible ofrecer un producto capaz de satisfacer los mercados más exigentes, tanto internos como externos, otorgando al productor interesantes expectativas de ingresos que justifican ampliamente los costos adicionales que este proceso implica.

En nuestro país, los resultados obtenidos con el secado a alta temperatura no han sido exitosos a nivel industrial, debido a la escasa experiencia que existe en relación con esta técnica y a que las recomendaciones que entregan los fabricantes son adecuadas para especies foráneas, cuyas características no siempre coinciden con las de las especies que crecen en Chile, o bien para condiciones operativas que tampoco corresponden con la realidad de la industria maderera nacional.

Como una forma de contribuir a resolver este problema, el presente informe da a conocer en detalle cuáles son las condiciones de secado más apropiadas para obtener madera de calidad, específicamente en el caso de la especie Pino Radiata.

\section{MATERIAL Y METODO}

Material experimental: El material utilizado en los ensayos es madera de Pino Radiata proveniente de bosques de la Octava Región. Se empleó madera aserrada con escuadrías de $25 \times 10 \mathrm{~mm}, 25 \times 125 \mathrm{~mm}, 50 \times 100 \mathrm{~mm}$ y $50 \times 200 \mathrm{~mm}$ y largos de 3000,3200 y $4000 \mathrm{~mm}$ y para los ensayos de laboratorio probetas de $25 \times 100 \mathrm{~mm}$ con largos de 3000 y $1000 \mathrm{~mm}$.

Diseño experimental: El diseño de los ensayos a nivel industrial se realizó considerando las variables más características del proceso de secado, a saber:

- Temperatura de bulbo seco

- Diferencia psicrométrica

- Reacondicionado

- Calentamiento

- Dimensión y espaciamiento de separadores

- Escuadría

- Peso sobre las pilas

- Ancho de las pilas

- Pretorcedura

- Humedad inicial

- Humedad final

Los resultados se midieron en términos de:

- Defectos de secado

- Humedad final de la carga

- Requerimientos energéticos y costos asociados

- Contracciones de la madera.

En lo que respecta a las experiencias de laboratorio, se consideraron como variables la humedad final e inicial y como resultado la temperatura de la madera y las curvas de secado.

\section{RESULTADOS}

Efecto de algunos parámetros del secado sobre la calidad de la madera secada:

- Efecto de la humedad final: La Figura 1 muestra el efecto que ejerce la humedad 
final sobre la torcedura dentro de un mismo programa de secado. Se aprecia que la torcedura aumenta al disminuir la humedad final, obteniéndose un $45 \%$ de las muestras en el grado 2 para la humedad final más alta y un $31 \%$ para la más baja.

- Efecto de la temperatura en seco: La Figura 2 muestra el efecto de la temperatura de bulbo seco sobre la torcedura en dos programas de secado diferentes manteniendo la misma diferencia psicrométrica. Se observa que en las muestras secadas a mayor temperatura de bulbo seco, los resultados medidos en términos de la torcedura producida son más positivos, obteniéndose un $58,5 \%$ de las muestras en el grado 2 para la temperatura de bulbo seco más alta y un $27,5 \%$ para la más baja.

- Efecto de la diferencia psicrométrica: La Figura 3 muestra la influencia de la diferencia psicrométrica sobre la torcedura en dos programas diferentes, manteniendo la misma temperatura de bulbo seco. Se aprecia que una mayor diferencia psicrométrica aumenta los defectos geométricos, encontrándose un $30 \%$ de las muestras en el grado 2 para una diferencia psicrométrica mayor un $66 \%$ para la diferencia psicrométrica menor.

- Efecto del peso sobre las pilas: La Figura 4 muestra la influencia que el peso sobre las pilas ejerce en la torcedura para un mismo programa de secado. Se observa que el peso mayor da mejores resultados en términos de la torcedura, encontrándose en el grado 2 un $82^{\circ} / \mathrm{o}$ de las muestras correspondientes al peso mayor y un $25 \%$ de las correspondientes al peso menor.

- Efecto de la pretorcedura: La Figura 5 muestra el efecto de la pretorcedura sobre la torcedura para un mismo programa de secado. Se puede apreciar que en el ensayo con pretorcedura antihoraria de las pilas hay un efecto de torcedura más bajo, tal como ocurrió en ensayos relizados con Pino elliotti $(9,12)$. Se obtuvo un $70 \%$ de las muestras en el grado 2 para este tipo de torcedura y un $44 \%$ para las pilas normales.

Efecto del reacondicionamiento: La Tabla 1 muestra la influencia del reacondicionado sobre los defectos evaluados cualitativamente para un mismo programa de secado. Se aprecia que el reacondicionado produce un mayor número de muestras defectuosas en cuanto a coloración, pero disminuye las grietas y rajaduras superficiales.

FIGURA 1.

EFECTO DE HUMEDAD FINAL (HF)
FIGURA 2

EFECTO DE TEMPERATURA DE BULBO SECO (TBS)

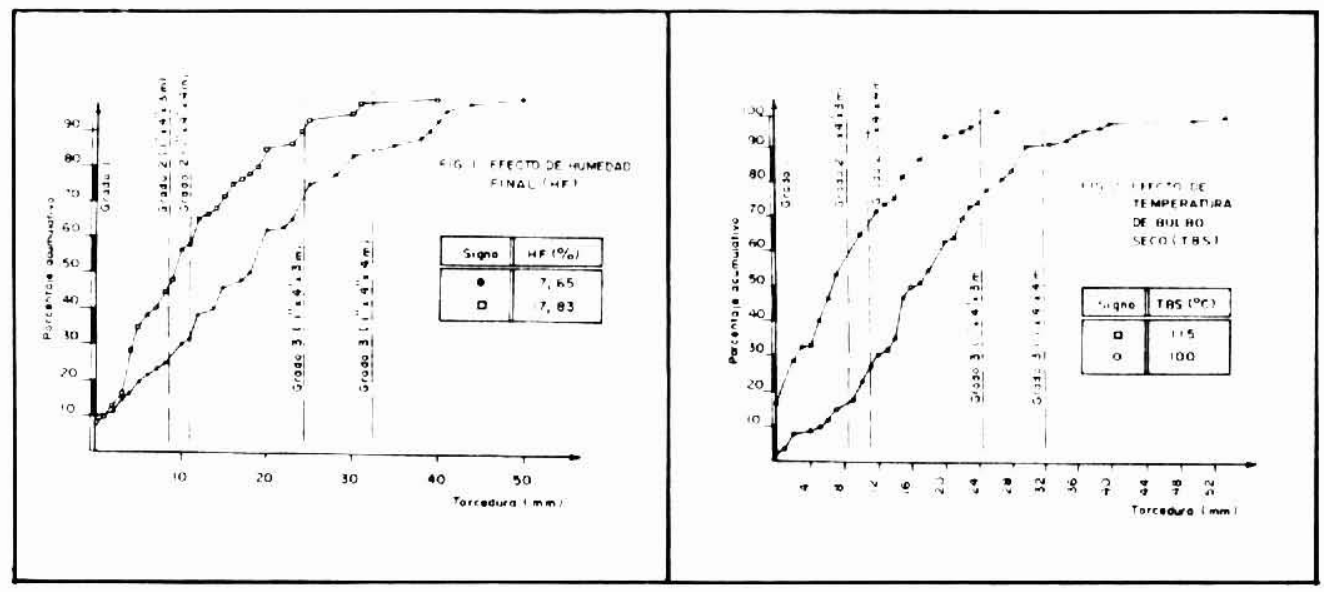


Efecto de la temperatura de bulbo seco: La Tabla 2 muestra el efecto de la temperatura de bulbo seco sobre los defectos evaluados cualitativamente, manteniendo la diferencia psicrométrica constante. Se puede apreciar que al aumentar la temperatura de bulbo seco se produce un aumento en el flujo de resina y aflojamiento de nudos, resultado similar al obtenido con Pino oregón (8).

FIGURA 3.

EFECTO DE DIFERENCIA SICROMETRICA (DS)
FIGURA 4.

EFECTO DEL PESO SOBRE LAS PILAS

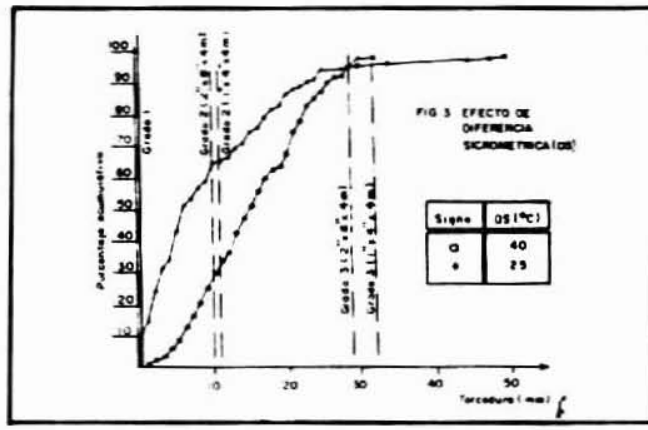

TABLA 1

EFECTO DEL REACONDICIONADO

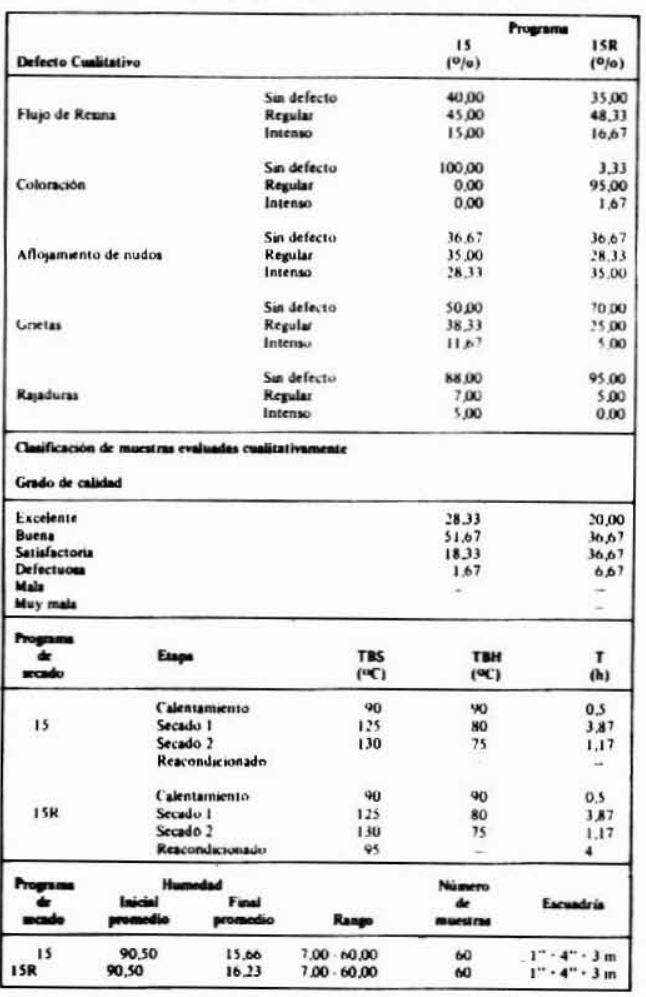

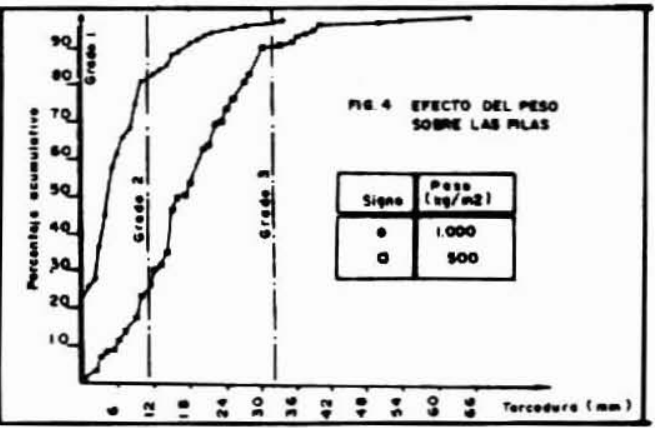

TABLA 2

EFECTO DE LA TEMPERATURA DE BULBO SECO

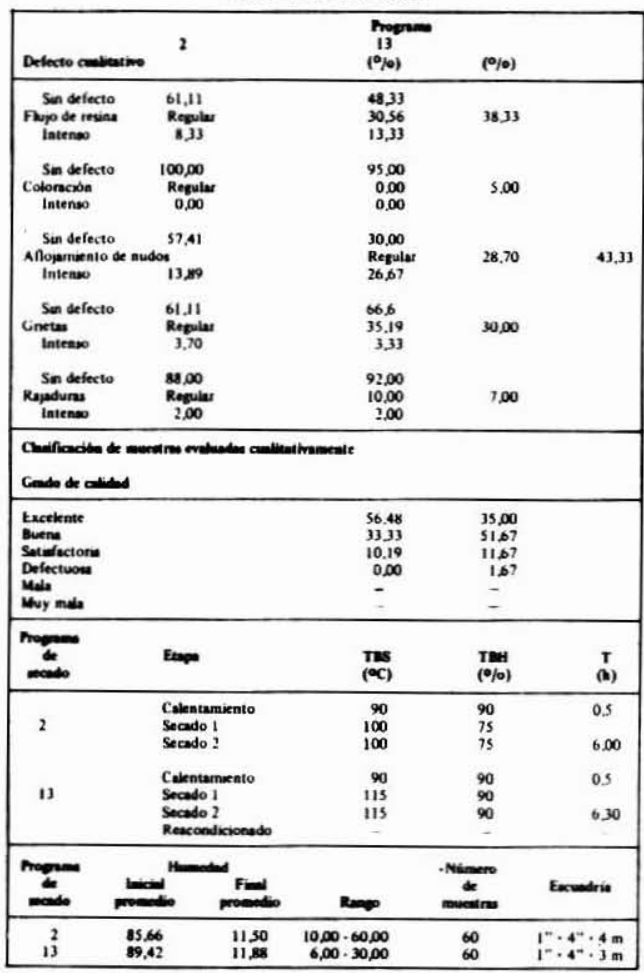


- Efecto de la humedad final: La Tabla 3 muestra el efecto de la humedad final sobre las tensiones para un mismo programa de secado. Se puede apreciar que las tensiones son menores al disminuir la humedad final, ya que junto con ésta disminuye el gradiente de humedad.

- Efecto de reacondicionamiento: La Tabla 4 muestra la influencia del reacondicionado sobre las tensiones para un mismo programa de secado. Se observa una significativa mejoría de las tensiones para los programas que contemplan un reacondicionado de cuatro horas. Esto se debe a la disminución del gradiente de humedad en el espesor de la tabla al aumentar la humedad superficial (15).

- Efecto de la temperatura de bulbo seco: La Tabla 4 muestra el efecto de la temperatura de bulbo seco sobre las tensiones, manteniendo la diferencia psicrométrica. Al comparar los ensayos sin reacondicionar, se aprecia que la alta temperatura aumenta sustancialmente las tensiones; sin embargo, esta diferencia se minimiza al reacondicionar ambos ensayos.

TABLA 3.

\section{EFECTO DE LA HUMEDAD FINAL}

\begin{tabular}{|c|c|c|c|c|}
\hline & & \multicolumn{3}{|c|}{ Programa } \\
\hline & & 13 & & 15 \\
\hline \multirow{4}{*}{\multicolumn{2}{|c|}{$\begin{array}{l}\text { Tensión promedio }(\% / 0) \\
\text { Rango }(\% / 0) \\
\text { Varianza }(\%) \\
\text { Humedad final promedio }(\%) \text {. }\end{array}$}} & 2,01 & & 11,45 \\
\hline & & $-3,70: 14,46$ & & $2,41: 21,10$ \\
\hline & & 5,28 & & 7,93 \\
\hline & & 13,55 & & 20,25 \\
\hline $\begin{array}{l}\text { Programa } \\
\text { de } \\
\text { secado }\end{array}$ & Etaps & $\begin{array}{l}\text { TBS } \\
\left({ }^{\circ} \mathrm{C}\right)\end{array}$ & $\begin{array}{l}\text { TBH } \\
\left({ }^{\circ} \mathrm{C}\right)\end{array}$ & $\begin{array}{c}\mathbf{T} \\
\text { (h) }\end{array}$ \\
\hline \multirow{4}{*}{13} & Calentamiento & 90 & 90 & 0,5 \\
\hline & Secado I & 115 & 90 & \\
\hline & Secado 2 & 115 & 90 & 6,3 \\
\hline & Reacondicionado & 95 & & 4 \\
\hline \multirow{4}{*}{15} & Calentamiento & 90 & 90 & 0,5 \\
\hline & Secado 1 & 125 & 80 & 3,83 \\
\hline & Secado 2 & 130 & 75 & 1,17 \\
\hline & Reacondicionado & 95 & & 4 \\
\hline
\end{tabular}

TABLA 4:

\section{EFECTO DE REACONDICIONADO Y TEMPERATURA DE BULBO SECO}

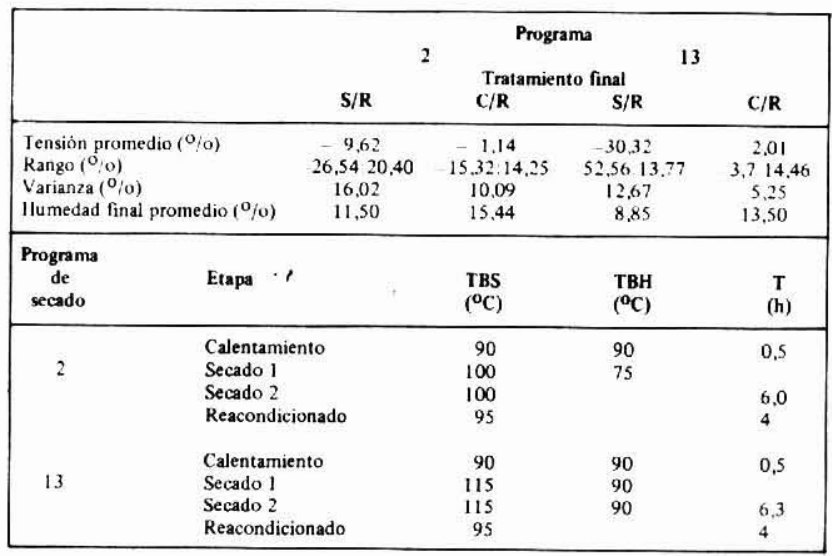


- Efecto de la saturación del aire: La Figura 6 muestra el efecto de la saturación del aire a medida que éste circula a través de las pilas. Se aprecia que la humedad final de la madera va aumentando mientras el aire fluye a lo ancho de la cámara, debido a que el potencial de secado del aire va disminuyendo al aumentar su humedad relativa como consecuencia de la captación de agua desde la madera.

- Efecto del ancho de las pilas: Las Figuras 7, 8 y 9 muestran la influencia del ancho de la pila sobre la distribución de la humedad final para un mismo programa de secado. En pilas más angostas se obtiene una distribución más uniforme para un valor medio similar, debido a que en este caso se produce una menor saturación del aire, tal como muestra la Figura 9.

FIGURA 5

EFECTO DE PRETORCEDURA

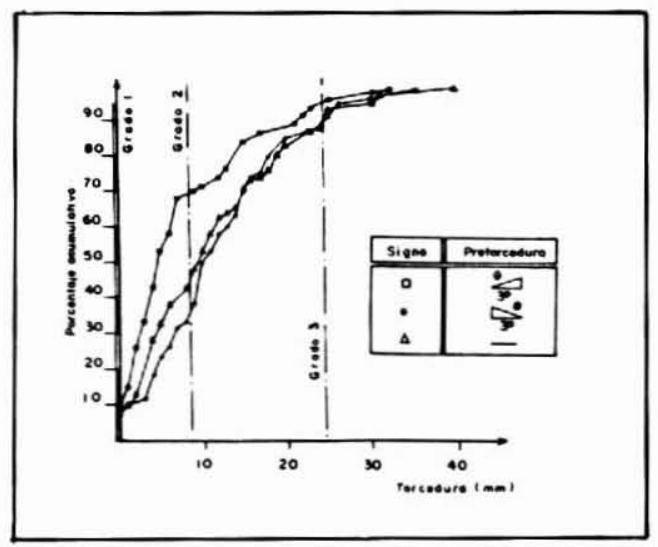

FIGURA 7

EFECTO DEL ANCHO DE PILA

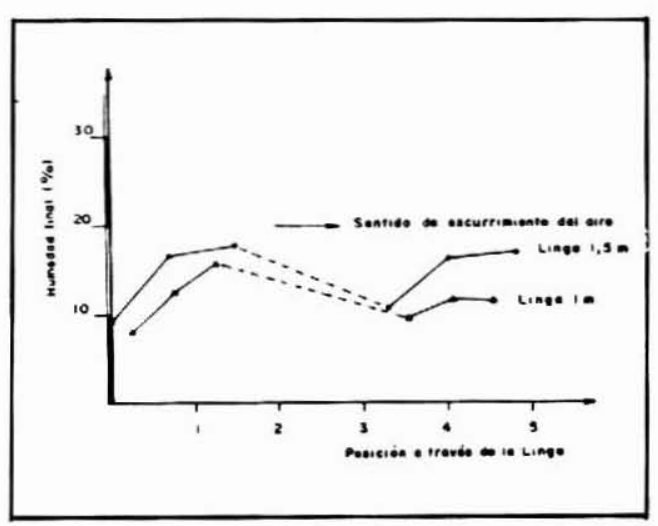

FIGURA 6

PROGRAMA NO 4

LINGAS 2-4, FILA 4

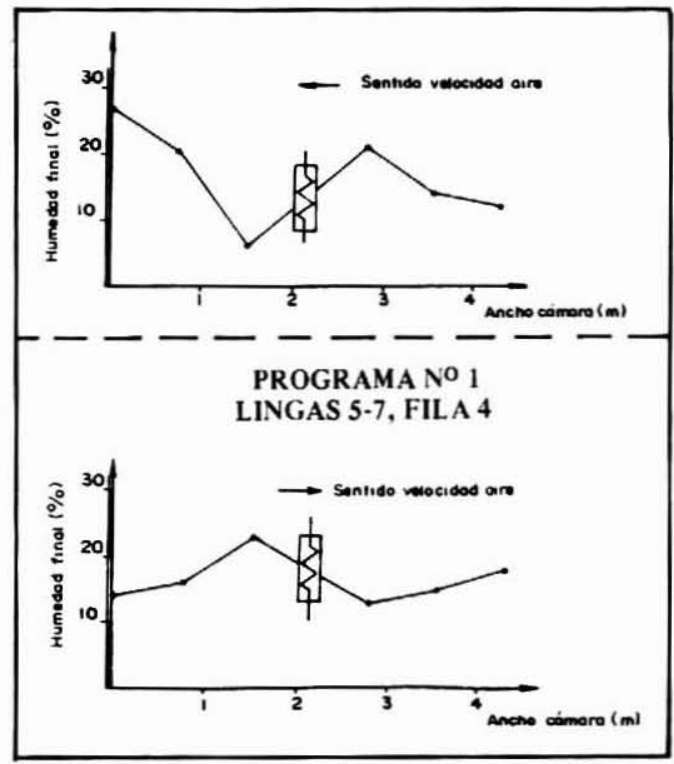

FIGURA 8

EFECTO DEL ANCHO DE PILA

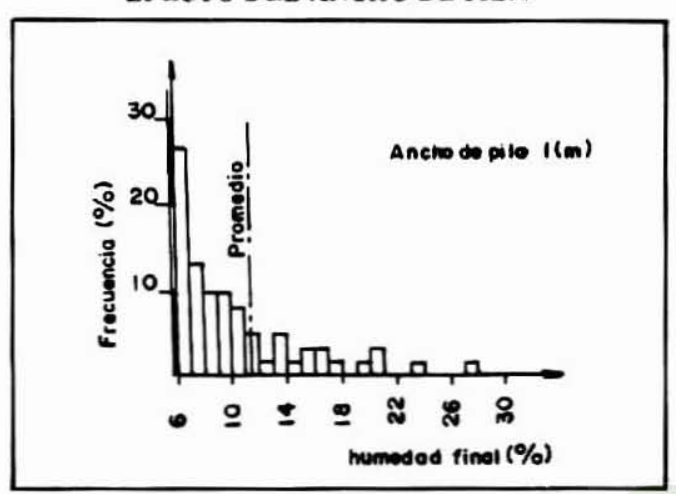


FIGURA 9

EFECTO DEL ANCHO DE PILA

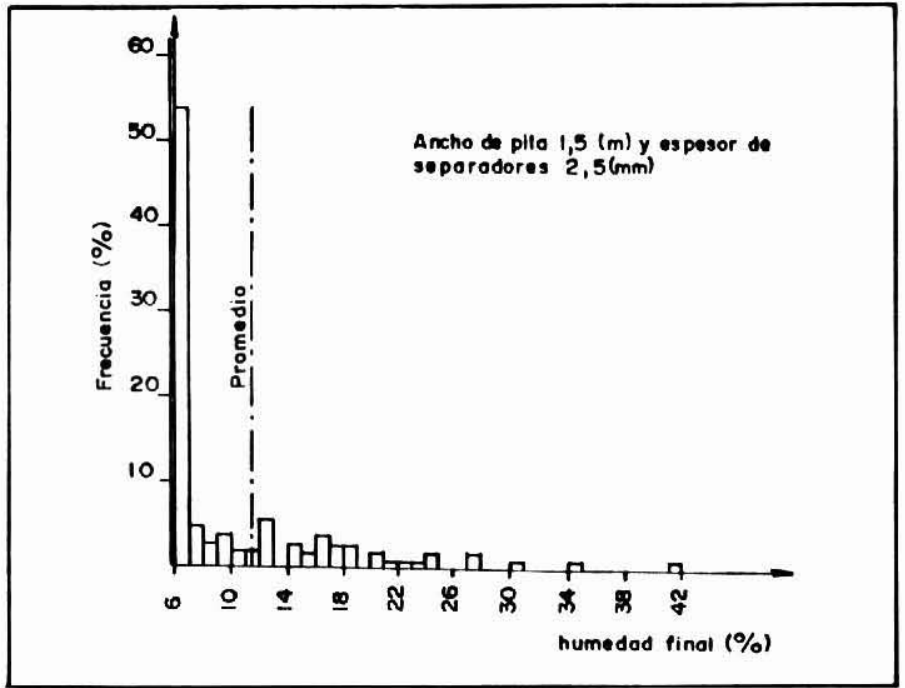

- Efecto del espesor de los separadores: Las Figuras 10 y 11 muestran el efecto del espesor de los separadores sobre la distribución de la humedad final para un mismo programa de secado. En el ensayo con separadores de $50 \mathrm{~mm}$ se obtiene una distribución bastante más uniforme con un valor medio similar.

Efecto del reacondicionado: Las Figuras 12 y 13 muestran la influencia del reacondicionado sobre la distribución de las humedades para un mismo programa de secado. Es evidente la mejoría de ésta para un ensayo con un reacondicionado posterior (10), produciéndose un aumento en el valor medio de la humedad de un $2 \%$ aproximadamente.

FIGURA 10

EFECTO DEL ESPESOR DE SEPARADORES
FIGURA 11

EFECTO DEL ESPESOR DE SEPARADORES
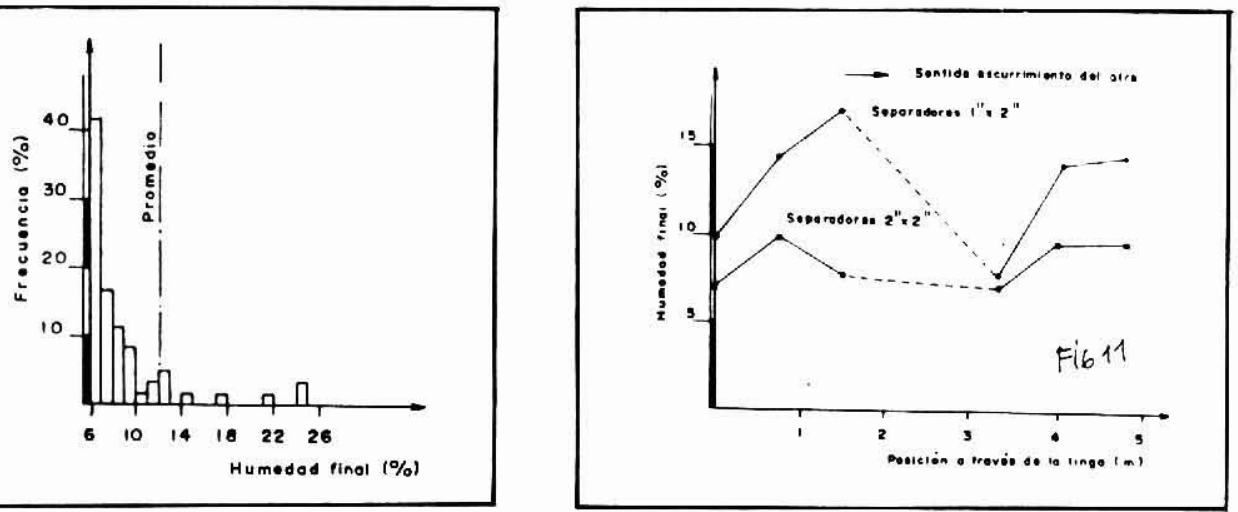
FIGURA 12

CARGA SIN TRATAMIENTO

DE REACONDICIONADO.

EFECTO DEL REACONDICIONADO

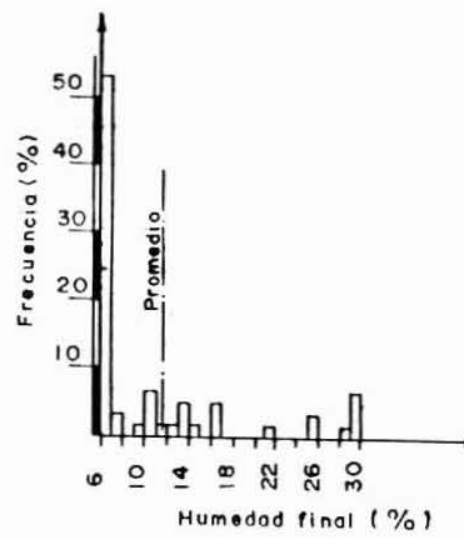

FIGURA 13

CARGA CON TRATAMIENTO

DE REACONDICIONADO.

EFECTO DEL REACONDICIONADO

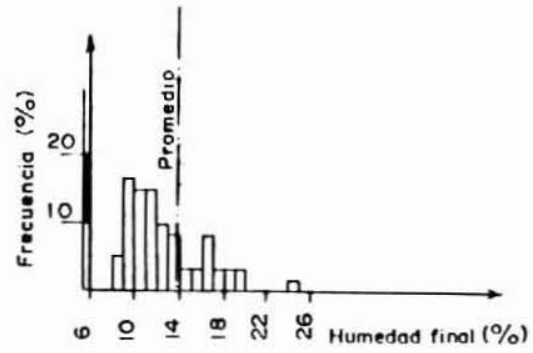

Efecto de la humedad final sobre la contracción de la madera

La Figura 14 muestra la correlación existente entre la humedad final y las contracciones tangencial y radial. Se puede observar que ambas aumentan a medida que disminuye la humedad final, de tal modo que a humedades cercanas al punto de saturación de la fibra los valores de tales contracciones son mínimos. Es notoria la diferencia entre la contracción radial y tangencial; esta última equivale aproximadamente al doble de la primera para los diferentes rangos de humedad $(5,15,18)$.

FIC;URA 14

CORRELACION CONTRACCIONES v

HUMEDAD FINAL

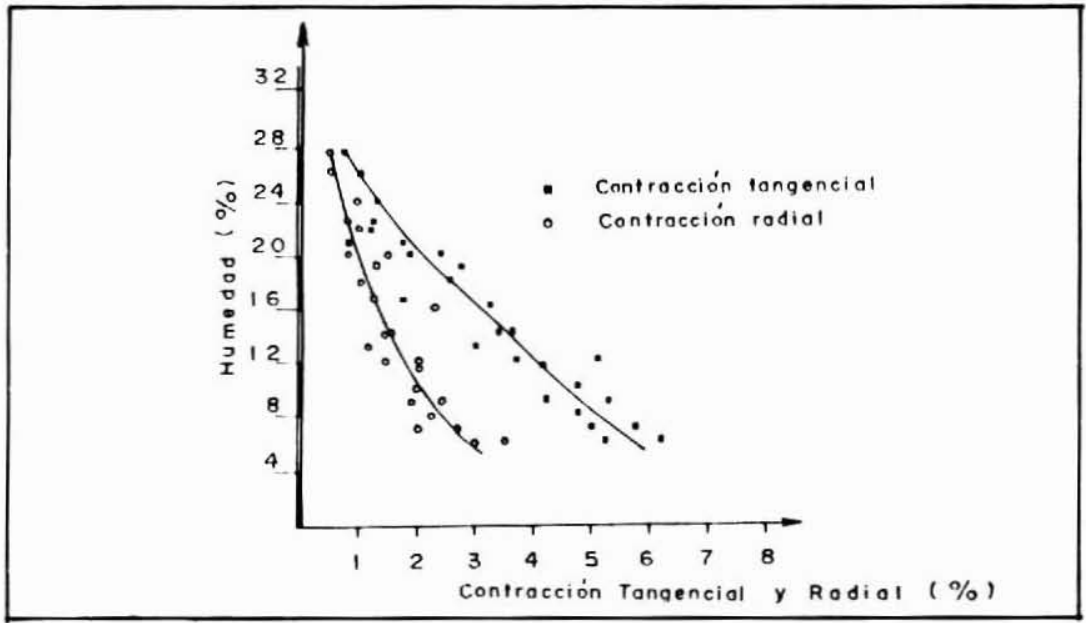




\section{Efecto de algunos parámetros de secado sobre los costos asociados al proceso}

- Efecto de la humedad final: La Tabla 5 muestra el efecto de la humedad final sobre los costos asociados de secado para un mismo programa. Se aprecia que el costo de extraer cada $\mathrm{kg}$ de agua es mayor mientras menor es la humedad final. En el caso ilustrado en la tabla, el costo adicional es de un $10 \%$ para una humedad final un $3,8 \%$ más baja.

- Efecto del reacondicionado: La Tabla 6 muestra la influencia del reacondicionado sobre los costos asociados de secado para un mismo programa. Se registra un aumento de un $43 \%$ del costo por unidad de masa de agua evaporada, como consecuencia del reacondicionado. Si comparamos el costo por unidad de volumen de madera secada, se obtiene un $53 \%$ de aumento.

\section{TABLA 5}

EFECTO DE LA HUMEDAD FINAL

\begin{tabular}{|c|c|c|}
\hline & \multicolumn{2}{|c|}{ Programa } \\
\hline & 17 & 18 \\
\hline Escuadría & $1 " \cdot 4 " \cdot 3 \mathrm{~m}$ & $1 " \cdot 4 " \cdot 3 \mathrm{~m}$ \\
\hline Volumen madera $\left(\mathrm{m}^{3}\right)$ & 21,67 & 21.67 \\
\hline H.medad inicial $(\%)$ & 90,50 & 97.30 \\
\hline Humedad final $(\% / \mathrm{o})$ & 14,20 & 17.80 \\
\hline Tiempo de secado $(\mathrm{h})$ & 6.50 & 5.50 \\
\hline Costo por unidad de volumen $\left(\$ / \mathrm{m}^{3}\right)$ & 174,77 & 166.28 \\
\hline Costo por unidad de masa $(\mathrm{S} / \mathrm{kg})$ & 0,50 & 0,45 \\
\hline \multicolumn{3}{|c|}{ Programa de secado } \\
\hline Etapa & TBS $\left({ }^{\circ} \mathrm{C}\right)$ & ТВ: $\left({ }^{\circ} \mathrm{C}\right)$ \\
\hline Calentamiento & 90 & 90 \\
\hline Secado 1 & 100 & 75 \\
\hline Secado 2 & 100 & 75 \\
\hline
\end{tabular}

\section{TABLA 6 \\ EFECTO DEL REACONDICIONADO}

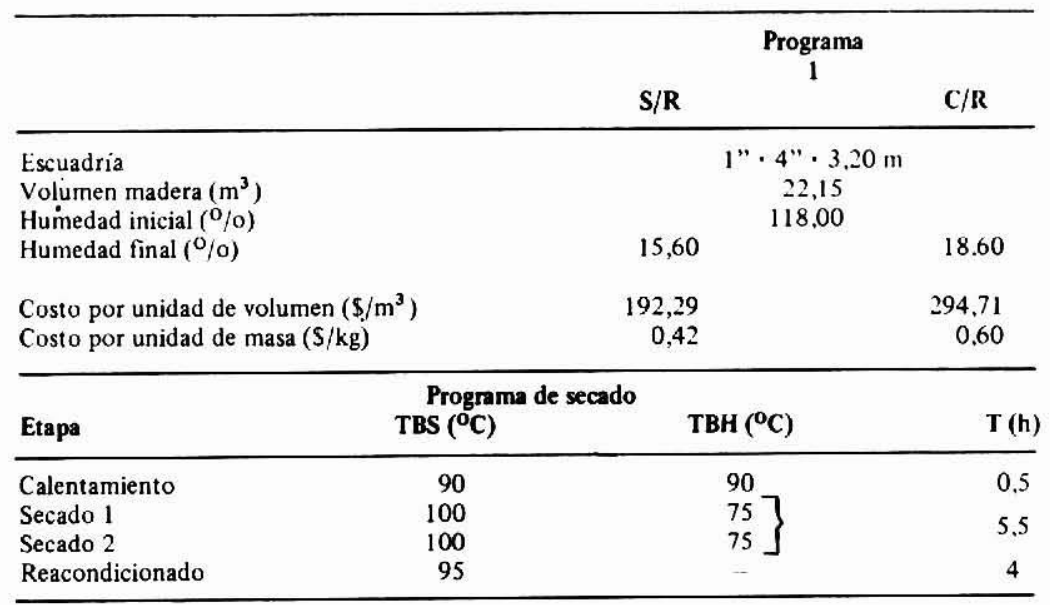


Efecto del ancho de las pilas y del espesor de los separadores: La Tabla 7 muestra el efecto del ancho de las pilas y del espesor de los separadores sobre los costos asociados de secado para un mismo programa. Tanto los costos por unidad de masa como por unidad de volumen son menores para el ensayo con pilas más angostas y con separadores de mayor espesor.

\section{Otros resultados experimentales}

Las Figuras 15 y 16 corresponden respectivamente a una curva y a una tasa de secado características. La forma de ambas curvas es similar a las encontradas para otros sólidos granulares (19) y otras especies de madera $(7,11,19$ y 20$)$.

La tasa de secado para el período de secado constante varía entre 0,92 y $2,38\left(\mathrm{~kg} / \mathrm{h} \mathrm{m}^{2}\right)$, con un valor medio de $1,60\left(\mathrm{~kg} / \mathrm{h} \mathrm{m}^{2}\right)$ para un mismo programa de secado. El punto a partir del cual la tasa deja de ser constante, o punto crítico, varía entre 17 y $45 \%$ de humedad.

Temperatura interna de la madera durante el proceso de secado: La Figura 17 permite apreciar la temperatura que alcanza la madera durante el proceso. Al comienzo, la temperatura es cercana a la temperatura de bulbo húmedo del aire, debido a que la madera se encuentra aún verde, de modo que se evapora una mayor cantidad de agua y se consume, en consecuencia,

FIGURA 15

MUESTRA NO 3 CURVA DE SECADO

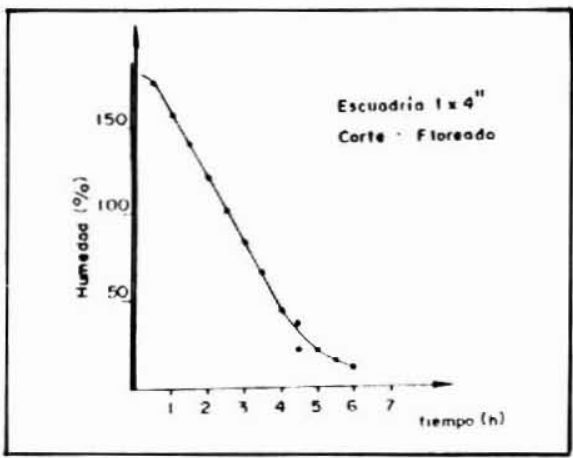

FIGURA 17

TEMPERATURA INTERNA DE MADERA DURANTE EL SECADO

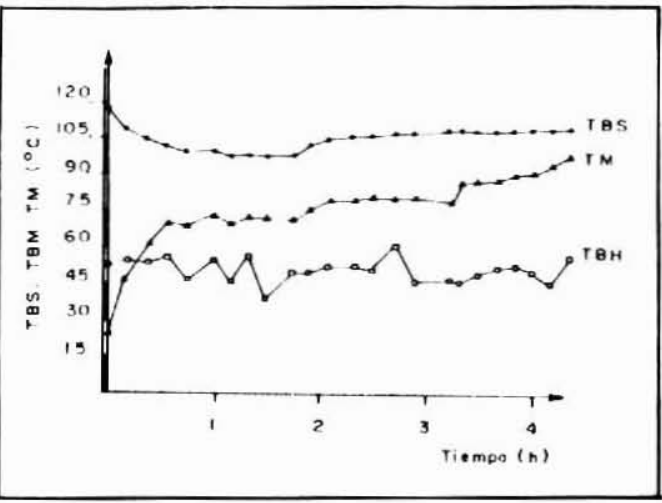

FIGURA 16

TASA DE SECADO

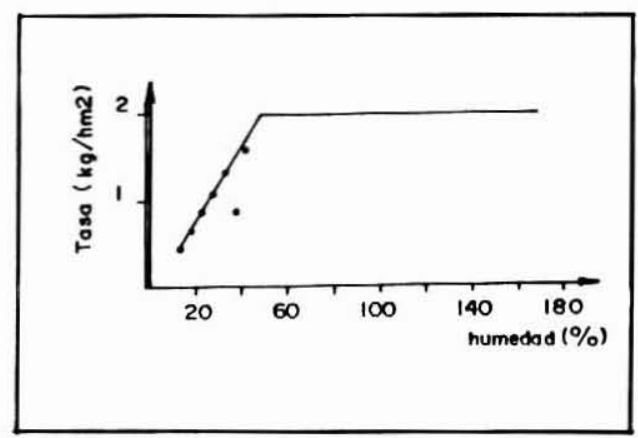

FIGURA 18

DIFERENCIA ENTRE TBS Y TEMPERATURA INTERIOR DE LA MADERA A LO LARGO DEL

SECADO

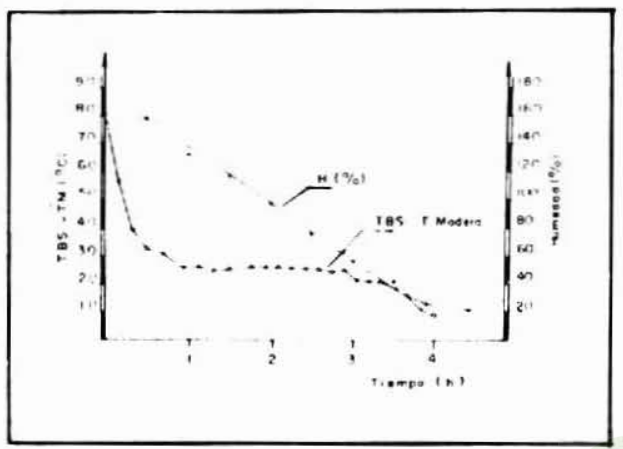


calor. En las etapas posteriores del proceso, la temperatura de la madera tiende a igualar la temperatura del bulbo seco del aire (16).

En la figura 18 se observa cómo varía la diferencia entre la temperatura de bulbo seco y la temperatura de la madera a medida que transcurre el secado. La diferencia es máxima cuando la madera se está calentando, posteriormente disminuye hasta un cierto valor y permanece constante mientras existe una relación lineal entre la humedad y el tiempo (tasa de secado constante); en la última etapa del proceso la diferencia de temperaturas se hace menor, ya que la temperatura de la madera tiende a la de bulbo seco, como se indicó anteriormente.

\section{TABLA 7 \\ EFECTO DEL ANCHO DE LAS PILAS \\ Y ESPESOR DE SEPARADORES}

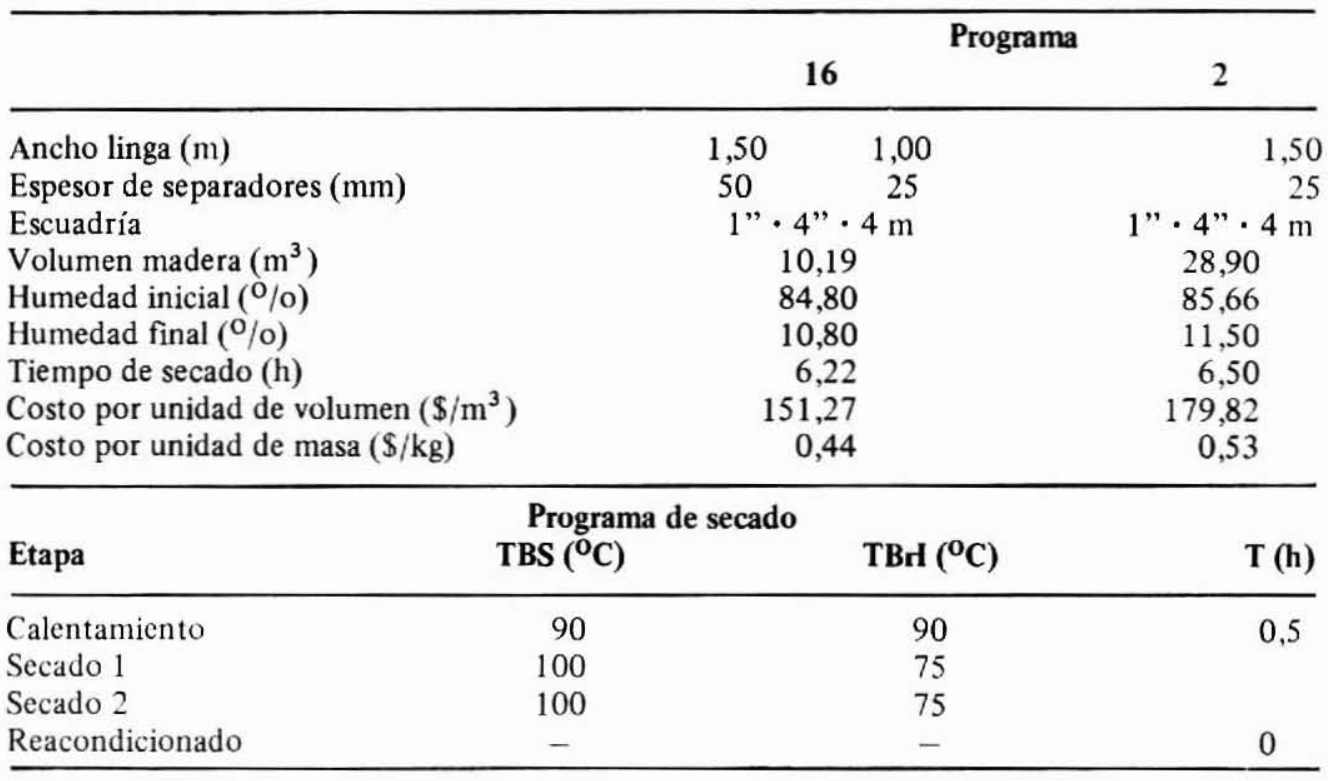

NOTA: El ensayo $\mathrm{N}^{\mathrm{O}} 16$ consistió de 4 pilas con separadores de $25 \mathrm{~mm}$ y ancho de $1,0 \mathrm{~m}$ y 4 pilas con separadores de $50 \mathrm{~mm}$ y ancho $1,5 \mathrm{~m}$.

\section{CONCLUSIONES Y RECOMENDACIONES}

\section{Preparación de la carga de secado:}

- Se recomienda construir las pilas de madera de $1 \mathrm{~m}$ de ancho y con separadores de $50 \mathrm{~mm}$ de espesor, colocados a una distancia de $0,60 \mathrm{~m}$ entre sí.

Es conveniente dar a las pilas una pretorcedura antihoraria de $3^{\circ}$.

- El peso que se coloca sobre las pilas debe aumentarse a $1000 \mathrm{~kg} / \mathrm{m}^{2}$.

\section{Programa de secado:}

- Es aconsejable elevar la temperatura de bulbo seco tanto como sea posible, manteniendo una diferencia psicrométrica baja.

La aplicación de tratamiento de reacondicionado depende de aspectos tales como uso 
posterior de la madera, coloración deseada y uniformidad de la humedad final de las piezas. Si se desea obtener madera libre de tensiones y con una humedad final pareja, es recomendable aplicarlo. En cambio, si es más importante contar con madera sin defectos de coloración, no debería aplicarse.

\section{Conclusiones complementarias:}

- La humedad final de la madera es uno de los factores que más influyen sobre los defectos de secado, de modo que el proceso no debe prolongarse hasta una humedad más baja que la exigida por el cliente. Al hacerlo, se incrementan innecesariamente los defectos geométricos y el costo.

- La humedad inicial no constituye una limitante demasiado significativa con respecto al tiempo de secado, ya que la velocidad de secado es directamente proporcional a la humedad inieial durante el período de velocidad de secado constante.

- Para efectos de mejorar la distribución de humedad la final, es recomendable instalar un sistema que permita invertir la circulación de aire cada cierto tiempo.

Las sobremedidas requeridas para secar madera hasta una humedad final de $15 \%$ son $1,4 \%$ en el espesor y $3,25 \%$ en el ancho, o viceversa, dependiendo del tipo de corte (floreado o cuarteado).

- El defecto geométrico de mayor importancia es la torcedura; la arqueadura y la encorvadura son de menor importancia y la acanaladura es despreciable, ya que la mayoría de las muestras no presentan el defecto.

$\mathrm{La}$ construcción de las pilas tiene gran influencia sobre la calidad de la madera secada. Por esta razón debe tenerse especial cuidado en el alineamiento entre separadores, entre los separadores y los cabezales ubicados entre pilas y bajo ellas y entre los cabezales y los pesos de hormigón.

Se recomienda no mezclar en una misma carga maderas de diferentes espesores ni tampoco madera recién aserrada con madera presecada al aire, ya que los tiempos de secado pueden variar notablemente.

\section{REFERENCIA BIBLIOGRAFICA}

1. MURUA, P. Contribución al estudio del secado natural de la madera de pino insigne, pp 29-39, Concepción (1969).

2. PECK, E. and Mc MILLEN, J. Lumber Seasoning, pp 116-119, Washington (1981). Agencia Internacional para el Desarrollo. Lab. Productos Forestales, USDA.

3. VILLIERE, A. Séchage des bois, Dunod, París, (1966).

4. ESTACION METEOROLOGICA BELLAVISTA. Universidad de Concepción, "Anuario Meteorológico 1981, Concepción (1981), pp 35.

5. SIAU, J.F. Flow in Wood, Syracuse University Press, New York (1971), pp 10-45.

6. KOCK, P. Process for Steam Straightening and Kiln Drying Lumber, Southern Lumberman, Vol (2), pp $10-13$ (1974).

7. KOCH, P. High-Temperature Drying of Southern Pine Timbers Lumber, and thick veneer, Southern Lumberman, Vol. (1), pp 11-14 (1974).

8. Sumi, H. y Mc Millen, J., High - Temperature Drying of Douglas- Fir Dimension Lumber, Forest Products Journal, Vol. (29), pp 25-32 (1979).

9. CHRISTENSEN, F.J. y GOUGH, D. Control of Drying Distortion in Framing From Young Plantation Slash Pine, Queensland (1975) Building Research, CSIRO. Report 44, Queensland Dept. of Foresty, pp 1-85. 
10. CHRISTENSEN, F.J. and MACKAY, J.F.G. The Future and Practical Application of High Temperature Seasoning of Plantation Softwoods, Queensland (1972). Australian Timber Industry Stabilisation Conference. pp 1-10.

11. KOLlmaNN, F.P. and COTE, W. Principles of Wood Science and Tecnology, Berlin, SpringerVerlag. (1968). V. 1, pp 60-110.

12. FRASER, H.R. Mill Solves Slash Pine Production Challenges. World Wood, 4:20-23 (1982).

13. BAMBER, R.K. and BURLEY, J. The Wood Properties of Radiata Pine, $1^{\mathrm{a}}$ Ed., pp 35-39, Londres (1983).

14. MACKAY, J.F. and RUMBALL, B.L. Drying of Distortion Prone Juvenile Core Radiata Pine for House Studs Australian Timber Journal, Vol. (6): 43-57 (1971).

15. INSTITUTO FORESTAL, Construcciones en madera. Santiago, Chile (1978). 10 fasc.

16. BLUHM, E. et al. El colapso en la madera y su reacondicionamiento, $23 \mathrm{p}$. Santiago, Chile. Instituto Forestal. Informe Técnico No 22.

17. ESTEVEZ, G. El secado de alta temperatura para madera aserrada de Pinus Radiata, Valdivia (1978). Universidad Austral de Chile, Centro Experimental Aserradero Vista Alegre, 100 p.

18. FOREST PRODUCTS LABORATORY FOR SERV. USDA. WOOD HANDBOOK, WASHINGTON (1955). Agriculture Handbook No 072. 528 p.

19. TREYBAL, R. Operaciones con Transferencia de Masa, $2^{\text {a }}$ Edición, Hispanoamericana S.A., Buenos Aires (1973) pp 667-682.

20. HILDEBRAND, R. Kiln Drying of Swan Timber, Alemania (1970).

21. KOCH, P. Suthern Pine Studs Can Be Dried And Steam-Strarghtened in 24 hours, Southern Luberman, (2723): 2-29 (1969).

22. SKAAR, C. Water in Wood, New York, Syracuse University Press (1972), pp 18.

23. PITTS, D. y SISSOM, L. Teoría y Problemas de Transferencia de Calor, $1^{\mathrm{a}}$ Ed., Bogotá, Ed. Mc Graw-Hill

23. PITTS, D. y SISSOM, L. Teoría y Problemas de Transferencia de Calor, $1^{\text {a }}$ Ed., Bogotá, Ed. Mc Graw-Hill Latinoamericana, S.A., (1979).

24. Villiere, A. Pertes de Chaleur Par Les Parois Des Se'choirs A'Bois Répercussions Techniques et Economiques, Québec (1969).

25. BACHRICH, J.L., Dry Kiln Handbook, H.A. Simins (International) Ltd., Vancouver (1980)

26. DE SAMPAIO, L.C. Perguntas E Respostas em Secagem De Madeiras, Sao Paulo (1981).

27. BOONE, R.S. An Introduction to High-Temperature Drying: Past Research Efforts and Definition of Terms and Procedures, (1979). In: Proceeding of a Symposium Held at New Albany, Indiana. USDA, pp 82.

28. MELO, R., Propiedades y Tratamientos de la Madera, Dirección de Investigaciones Tecnológicas y Asistencia Técnica, Concepción (1981).

29. RIUS, G. y VALENZUELA, L. Estudio de Permeabilidad de Gases y Líquidos en madera de Pino insigne (Pinus radiata D. Don), Universidad del Bío Bío, Departamento de Maderas, Concepción (1981). Tomo I, 49 p. Tomo II, 40 p.

30. OliVeros, J.F. Secamiento artificial de durmientes de Pino insigne, Universidad Técnica del Estado, Sede Concepción, Departamento de Maderas, Concepción (1969). pp 38.

31. INSTITUTO NACIONAL DE NORMALIZACION. Madera Aserrada de Pino insigne (Pinus radiata D. Don). Clasificación por aspecto. NCh of 79, Santiago (1979).

32. SALGADO, S. Aplicación y análisis de tres programas de secado artificial en madera de Lenga (Nothofagus pumilio (Poepp. et Ende) Krasser). (1974) 70 p.

33. ENDESA. Plan de desarrollo de ejecutivos, ciclo de charlas sobre política tarifaria. Santiago (1980). 